\title{
Evaluación de la eficiencia energética del cuesco de cacay (Caryodendron orinocense)
}

\section{Evaluation of the energy efficiency of the cuesco de cacay (Caryodendron orinocense)}

\section{Avaliação da eficiência energética do cuesco de cacay (Caryodendron \\ orinocense)}

\begin{abstract}
González Álvarez Angie Carolina1, Rodríguez Jiménez Luis Alejandro", Laguna Chacón Jaime Ricardo ${ }^{2}$ y López Muñoz Luis Gilberto ${ }^{2}$

${ }^{1}$ Ingenieros Agroindustriales, Universidad de los Llanos y

${ }^{2}$ Ingenieros Agroindustriales, MSc, Docentes Universidad de los Llanos

llopezm@unillanos.edu.co
\end{abstract}

Recibido 14 de septiembre 2020 aceptado 16 de noviembre 2020

\section{RESUMEN}

En el presente proyecto se evaluó el poder calorífico del cuesco de Cacay (Caryodendron orinocense) proveniente del municipio de Villavicencio, determinando el poder calorífico del cuesco de cacay a diferentes muestras que fueron sometidas a dos tipos de secado y llevadas a dos tamaños de partícula. Para esto se recolectaron cuescos de cacay provenientes de una empresa dedicada a la extracción del aceite de su nuez, se les retiraron las impurezas, se molieron y se tamizaron. A continuación, se sometieron a los tipos de secado, unas muestras se expusieron a secado por horno durante 12 horas y a $102^{\circ} \mathrm{C}$, y otras muestras se secaron al sol durante dos días con temperaturas cercanas a los $32^{\circ} \mathrm{C}$, condiciones naturales de la ciudad de Villavicencio, una vez preparadas las muestras se determinó su poder calorífico por medio de una bomba calorimétrica. Así mismo, se estimó el consumo energético de cada uno de los pretratamientos a los que fueron sometidas las muestras con el fin de establecer un balance energético, determinando así cuales pretratamientos son justificables para optimizar su desempeño energético, teniendo como resultados valores de poder calorífico superiores comprendidos entre los 4189 y $4629 \mathrm{kcal} / \mathrm{kg}$, con diferencias energéticas 
significativas entre todos los tratamientos frente al blanco, encontrando que la muestra con mayor tamaño de partícula y secado artificial se presentó como la de mayor diferencia significativa de acuerdo al análisis de las varianzas (ANOVA), sin embargo se determinó que la muestra con mayor tamaño de partícula y secado solar, posee los pretratamientos más eficientes, en cuanto a consumo de energía, indicando que se puede alcanzar un poder calorífico superior al de otras biomasas residuales.

Palabras clave: Biomasa residual, biocombustible, consumo energético, poder calorífico.

\section{ABSTRACT}

In the present project, the calorific value of the cacay shell (Caryodendron orinocense) from the municipality of Villavicencio was evaluated, determining the calorific power of the cacay shell to different samples that were put through to two types of drying and taken to two particle sizes. To do this, cacay shell was collected from a company dedicated to the extraction of the oil from its nut; the impurities were removed, ground and sieved. Then, they were dried by two methods, some samples were exposed to drying by oven for 12 hours and at $102^{\circ} \mathrm{C}$, and other samples were dried in the sun for two days with temperatures close to $32^{\circ} \mathrm{C}$, natural conditions of the city of Villavicencio, once the samples were prepared, their calorific value was determined by means of a calorimetric pump. Likewise, the energy consumption of each of the pretreatments to which the samples were subjected was estimated in order to establish an energy balance, thus determining which pretreatments are justifiable to optimize their energy performance, having as a result higher calorific value values included between 4189 and $4629 \mathrm{kcal} / \mathrm{kg}$, with significant energy differences between all the treatments compared to the target, finding that the big size particle and oven drying was presented as having the greatest significant difference according to the analysis of the variances (ANOVA), however, it was determined that the big size particle and sun drying, has the most efficient pretreatments, in terms of energy consumption, indicating that a higher calorific value can be reached than other residual biomass. 
Keywords: Calorific power, residual biomass, biofuel, energy consumption.

\section{RESUMO}

No presente projeto, avaliou-se o poder calorífico da casca de cacay (Caryodendron orinocense) do município de Villavicencio, determinando o poder calorífico da casca de cacay para diferentes amostras que foram submetidas a dois tipos de secagem e levadas para dois tamanhos de partículas. Para fazer isso, a concha cacay foi coletada de uma empresa dedicada à extração do óleo de sua castanha; as impurezas foram removidas, moídas e peneiradas. Em seguida, eles foram secados por dois métodos, algumas amostras foram expostas a secagem por estufa por 12 horas e a $102^{\circ} \mathrm{C}$, e outras amostras foram secas ao sol por dois dias com temperaturas próximas a $32^{\circ} \mathrm{C}$, condições naturais da cidade. de Villavicencio, uma vez preparadas as amostras, seu poder calorífico foi determinado por meio de uma bomba calorimétrica. Da mesma forma, o consumo de energia de cada um dos prétratamentos aos quais as amostras foram submetidas foi estimado para estabelecer um balanço energético, determinando quais tratamentos prévios são justificáveis para otimizar seu desempenho energético, tendo como resultado valores de poder calorífico superior incluídos entre 4189 e $4629 \mathrm{kcal} / \mathrm{kg}$, com diferenças significativas de energia entre todos os tratamentos em relação à meta, constatando que a partícula de grande tamanho e a secagem em estufa apresentaram a maior diferença significativa de acordo com a análise das variâncias (ANOVA), entretanto, foi determinado que a partícula de grande tamanho e a secagem ao sol têm os prétratamentos mais eficientes, em termos de consumo de energia, indicando que um valor calórico mais alto pode ser alcançado do que outras biomassas residuais.

Palavras-chave: Potência calórica, biomassa residual, biocombustível, consumo de energia.

\section{INTRODUCCIÓN}

Actualmente, el uso de combustibles tradicionales no renovables ha generado un impacto negativo en el ambiente y a medida que se siguen utilizando de manera indiscriminada provocan un agotamiento de los recursos, esta situación afecta a 
todos los seres vivos de manera negativa, ya que la obtención de estos combustibles implica el deterioro de los diferentes ecosistemas. Es por esto que durante los últimos años se han investigado fuentes alternativas para la obtención de energía que sean renovables, más económicas, menos contaminantes y que puedan suplir la creciente demanda representada en un aumento poblacional que ve satisfechas sus necesidades energéticas mediante combustibles fósiles cada vez más escasos y altamente contaminantes.

Hoy en día, de acuerdo a Durango y Oquendo, (2016) el uso de la biomasa como fuente de energía ha demostrado ser una alternativa interesante por ser un recurso renovable, de bajo costo y amigable con el medio ambiente. Una ventaja adicional de la biomasa como sustituto de combustibles fósiles se encuentra en que esta presenta, según Herrera y Camargo, (2017) un balance neutro en el ciclo del dióxido de carbono, ya que durante el crecimiento de la planta esta absorbe $\mathrm{CO}_{2}$ y lo transforma en productos como la celulosa y hemicelulosa componentes de la biomasa, por lo tanto mediante la combustión no se introducen cantidades adicionales de $\mathrm{CO}_{2}$ como si ocurre durante la combustión de los materiales derivados del petróleo y del carbón.

Paralelamente la acumulación de la biomasa resultante de la industrialización de algunos productos se ha transformado en una problemática cada vez más preocupante, debido a que esta también puede generar un impacto negativo en el equilibrio del medio ambiente y a medida que la demanda de dichos productos aumenta, se produce una mayor cantidad de residuos, que al no ser aprovechados generan problemas tanto higiénico-ambientales como económicos, según Peñaranda et al., (2017) en el caso de Colombia durante el procesamiento de productos como: café, palma de aceite, caña de azúcar y panelera, maíz, arroz, banano y plátano se producen alrededor de 71.943.813 Ton/año de residuos que en la gran mayoría de los casos son incinerados o llevados a rellenos sanitarios.

El cacay (Caryodendron orinocense) es un fruto promisorio de la región de la Orinoquia colombiana por las propiedades cosméticas y alimenticias encontradas en su nuez, según el periódico El Tiempo, (2017) el departamento del Meta posee 
cultivos en forma silvestre capaces de generar unas 20.000 toneladas de aceite y cultivos de aproximadamente 57 hectáreas en Puerto Lleras y 120 hectáreas en Puerto Gaitán. Además de reportes publicados por la Cámara de Comercio de Bogotá, (2017) donde se informa que con la ayuda de ONG's y algunas Gobernaciones, se han distribuido árboles de cacay a diferentes familias permitiendo contar aproximadamente con 200 hectáreas de cultivo y 417 hectáreas que empresas privadas han sembrado en Puerto Gaitán, adicional a estos cultivos en el departamento del Meta, el portal web CONTEXTOGANADERO, (2013) registra una siembra en el departamento del Vichada de 100 hectáreas, impulsando de esta manera un crecimiento de este cultivo en el cual la densidad promedio es de 100 a 143 árboles por hectárea con un rendimiento cercano a los $250 \mathrm{~kg}$ de fruto por árbol anual, lo que permite estimar una producción de cuesco por hectárea de $11.666,2 \mathrm{~kg}$ por año. De igual manera PROCOLOMBIA, (2014) reporta demandas de aceite de cacay equivalentes a la producción de 3.900 hectáreas cultivadas, haciendo evidente la acumulación de este tipo de biomasa la cual carece de valor agregado.

Teniendo en cuenta lo anterior, esta investigación pretende determinar la eficiencia energética del cuesco del cacay y de esta manera dar un valor agregado a este residuo que ocupa aproximadamente el $33 \%$ de la masa del fruto; aunque actualmente el establecimiento del cultivo de cacay se encuentra en sus fases iniciales, este es un cultivo que se proyecta con gran crecimiento debido a las propiedades benéficas de la nuez y su alta demanda en el mercado cosmético, generando un elevado volumen de residuos que no presentan ningún proceso de transformación ocasionando un problema ambiental debido al mal manejo de los residuos sólidos y presentándose como una alternativa para la obtención de energía.

Por lo tanto, se presenta la siguiente pregunta de investigación: ¿El poder calorífico del cuesco de cacay será suficiente para sustituir otras biomasas residuales como fuente de energía calórica y en que afecta emplear pretratamientos como secado y reducción de tamaño de partícula en su potencial energético? 
Por esto se busca evaluar la eficiencia energética del cuesco del cacay (Caryodendron orinocense) en función del tipo de secado y tamaño de partícula mediante un proceso de combustión adiabático, estimando el consumo energético requerido para los procesos de molienda y secado necesarios para el establecimiento de las variables objeto de estudio, y así determinar el poder calorífico del cuesco del cacay en función del tipo de secado y tamaño de partícula por medio de una bomba calorimétrica y por ultimo establecer un análisis comparativo de los resultados obtenidos del poder calorífico del cuesco del cacay frente a los datos registrados de otras biomasas residuales (bagazo de caña de azúcar, cascarilla de arroz y cuesco de palma de aceite).

\section{METODOLOGÍA}

\section{Tipo de estudio}

La investigación es de tipo experimental ya que se basa en la observación, medición, análisis e interpretación de variables que intervienen en el desarrollo del proyecto.

\section{Diseño experimental y análisis estadístico}

Para un adecuado análisis de los datos se realizó un diseño experimental completamente al azar en donde se establecieron ensayos con dos tipos de secado $y$ dos intervalos de tamaño de partícula obteniendo un experimento factorial $2^{2}$, generando cuatro tratamientos.Para asegurar la cantidad de datos suficientes y un adecuado análisis de los mismos se hicieron ensayos por triplicado para cada uno de los tratamientos donde las variables independientes son el tipo de secado y el tamaño de partícula, así mismo la variable de respuesta es el poder calorífico como se indica en la siguiente tabla.

Para el análisis de los datos se utilizó el software estadístico $R$, con el objetivo de analizar correctamente los datos y así determinar si las variaciones del tipo de secado y tamaño de partícula afecta el poder calorífico del cuesco del cacay y que tan significativa resulta ser. 
Tabla 1. Diseño experimental factorial $2^{2}$

\begin{tabular}{cc}
\hline Factores & Niveles \\
\hline Tipo de Secado & Solar \\
& Horno \\
Tamaño de partícula* $^{*}$ & $2.36-0.25 \mathrm{~mm}$ \\
& $\leq 0.25 \mathrm{~mm}$ \\
\hline
\end{tabular}

Fuente: Los autores

\section{Obtención de la materia prima y adecuación}

El cuesco de cacay (Caryodendron orinocense K.) se obtuvo de cultivos ubicados en el municipio de Puerto Gaitán, la materia prima se sometió a un proceso de limpieza donde se retiró cualquier tipo de suciedad y luego se las partículas fueron afinadas mediante un proceso de tamizado con las aperturas que se indican en la Tabla 2, en el juego de tamices se incluyó un tamiz numero 60 con la finalidad de obtener partículas finas que no superaran un diámetro mayor a $1 \mathrm{~mm}$ para obtener un rango más amplio entre los diferentes diámetros.

Tabla 2. Diámetro del Juego de tamices utilizado en el cuesco de cacay

\begin{tabular}{cccc}
\hline Número de tamiz & $\begin{array}{c}\text { Apertura del } \\
\text { tamiz }(\mathbf{m m})\end{array}$ & $\begin{array}{c}\text { Intervalo } \\
(\mathbf{m m})\end{array}$ & $\begin{array}{c}\text { Diámetro promedio } \\
(\mathbf{m m})\end{array}$ \\
\hline $3 / 8$ in & 9.5 & $>9.5$ & 9.5 \\
6 & 3.35 & $9.5-3.35$ & 6.425 \\
7 & 2.80 & $3.35-2.80$ & 3.075 \\
8 & 2.36 & $2.80-2.36$ & 2.580 \\
60 & 0.25 & $2.36-0.25$ & 1.305 \\
Fondo & Fondo & $<0.25$ & 0.125 \\
\hline
\end{tabular}

Fuente: Los autores 


\section{Molienda y tamizado del cuesco del cacay}

El material tamizado fue sometido a un proceso de reducción de tamaño por medio de un molino de discos eléctrico de marca Victoria, equipado con un motor de 0.75 $\mathrm{Kw}$ de potencia, y a un estudio granulométrico para identificar los tamaños de partícula de mayor proporción y seleccionar las fracciones objeto de estudio. Una vez obtenidos los tamaños de partícula, las muestras se denominaron como se indica en la tabla 3 y se procedió a estimar la energía empleada para reducir el tamaño de partícula de acuerdo a la ecuación de Rittinger que se observa en la ecuación 1 (Gutiérrez y Ulloa, 2018).

Tabla 3. Tamaños de partícula

\begin{tabular}{cc}
\hline Nombre de la muestra & $\begin{array}{c}\text { Tamaño de partícula } \\
(\mathbf{m m})\end{array}$ \\
\hline T1 & $\leq 0.25$ \\
T2 & $2.36-0.25$ \\
T3 & $\leq 0.25$ \\
T4 & $2.36-0.25$ \\
\hline
\end{tabular}

Fuente: Los autores

Ecuación 1 Ecuación de Rittinger

$$
E=K_{R}\left(\frac{1}{d 2}-\frac{1}{d 1}\right)
$$

Dónde:

$\mathrm{E}=$ potencia $(\mathrm{kW}) /$ alimentación $(\mathrm{kg} / \mathrm{H})$

$\mathrm{KR}=$ constante de Rittinger (Hp.H.in/ton)

$\mathrm{d} 1=$ tamaño inicial $(\mathrm{mm})$

$\mathrm{d} 2=$ tamaño final $(\mathrm{mm})$ 


\section{Secado}

Las muestras T3 y T4 fueron sometidas a un proceso de secado solar en condiciones ambientales de la ciudad de Villavicencio por un tiempo establecido de 8 horas por cada día durante dos días, estas condiciones de tiempo fueron registradas con el fin de tener datos más precisos. Así mismo, las muestras T1 y T2 fueron sometidas a un proceso de secado al horno con una temperatura de $102^{\circ} \mathrm{C}$ durante 12 horas, en la Tabla 4 se muestra los tipos de secado utilizados para cada muestra y en las Figuras 1 y 2 se evidencia el montaje para cada tipo de secado.

Tabla 4. Variables evaluadas para medir la eficiencia energética

\begin{tabular}{ccc}
\hline Nombre de la muestra & Tipo de secado & $\begin{array}{c}\text { Tamaño de partícula } \\
\mathbf{( m m})\end{array}$ \\
\hline T1 & Horno & $\leq 0.25$ \\
T2 & Horno & $2.36-0.25$ \\
T3 & Sol & $\leq 0.25$ \\
T4 & Sol & $2.36-0.25$ \\
\hline
\end{tabular}

Fuente: Los autores

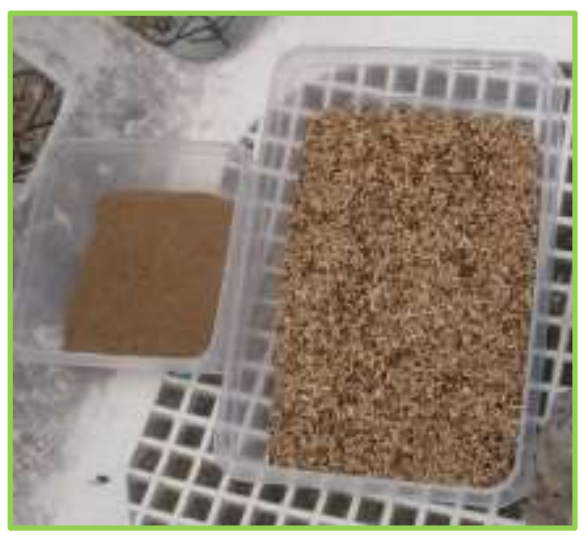

Figura 1. Secado al sol

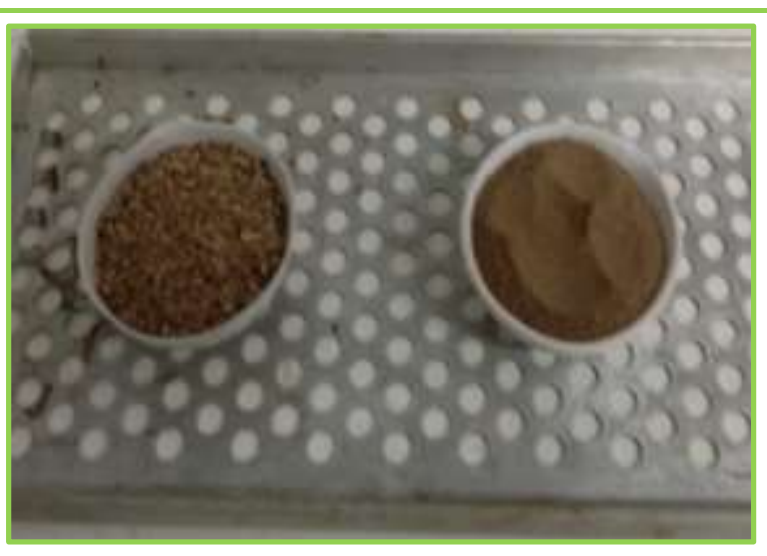

Figura 2. Secado al horno

Posteriormente se estimó el gasto energético empleado durante el proceso de secado de las muestras (T1 y T2), para esto se tomó en cuenta el consumo de 
energía del horno y el tiempo que demore el proceso de secado de la siguiente manera. Consumo energético $(\mathrm{Kcal} / \mathrm{H}) \times$ tiempo de secado $(\mathrm{H})=$ consumo energético total (Kcal).

De acuerdo al diseño experimental se obtuvo las muestras con las variables identificadas en la Tabla 4, permitiendo establecer las condiciones de operación de cada una de las muestras resultado de los pretratamientos.

\section{Determinación del poder calorífico}

El poder calorífico de las muestras (T1, T2, T3 y T4) se determinó en una bomba calorimétrica modelo 1341 de la marca Parr que se muestra en la ilustración 4, de acuerdo a la norma ASTM D-5865-04 (2004), cuyos montajes se evidencian en las Figuras 3 y 4 , esta prueba se realizó en los laboratorios de nutrición animal del Instituto de Acuicultura de los Llanos (IALL) de la Universidad de los Llanos.

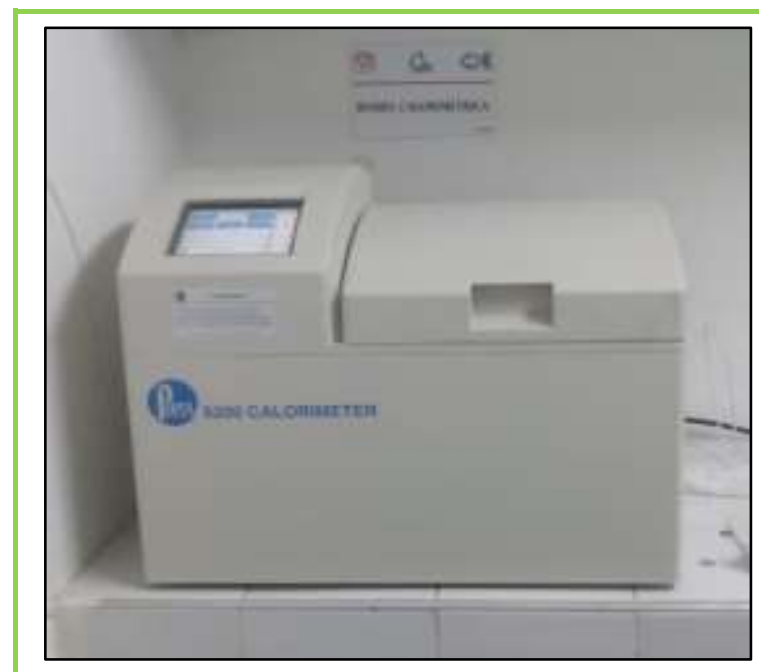

Figura 3. Bomba calorimétrica

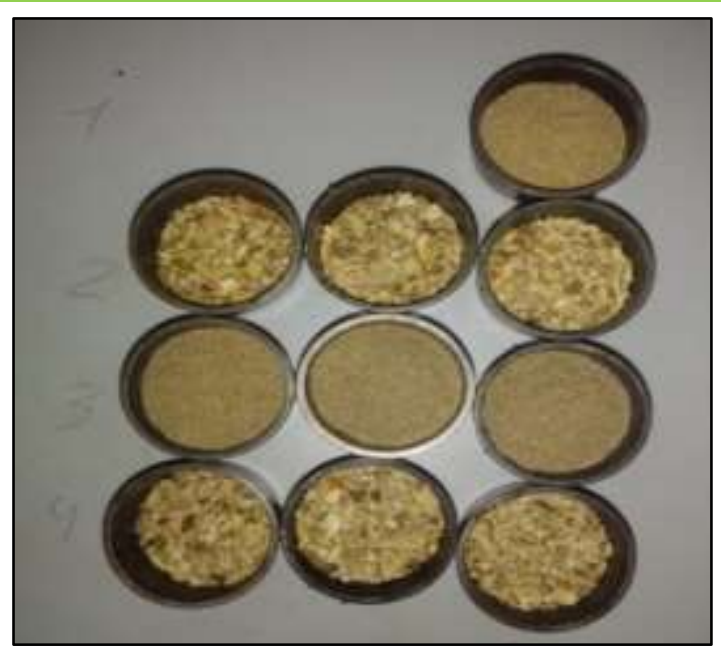

Figura 4. Muestras Para Bomba Calorimétrica

Adicional a la determinación del poder calorífico de las muestras previamente tratadas y objeto del análisis experimental, se determinó el potencial energético del cuesco sin ningún pretratamiento adicional al que se somete para la extracción de la nuez donde alcanza un tamaño de partícula promedio de $10.5 \mathrm{~mm}$ con formas irregulares como se observa en la Figura 5, esto con el fin de obtener un dato blanco 
o testigo que funcione como punto de referencia o patrón que permita establecer una comparación con los resultados obtenidos, de igual manera este análisis se realizó por triplicado.

\section{RESULTADOS Y DISCUSIÓN}

Previo a la realización de los pretratamientos al material, se llevó a cabo una limpieza y un proceso de tamizaje con el fin de eliminar cualquier material extraño e identificar el tamaño de partícula inicial, dando como resultado un tamaño de partícula inicial comprendido entre 10-11 mm aproximadamente, teniendo en cuenta que estas partículas se obtienen como producto de la extracción de su nuez.

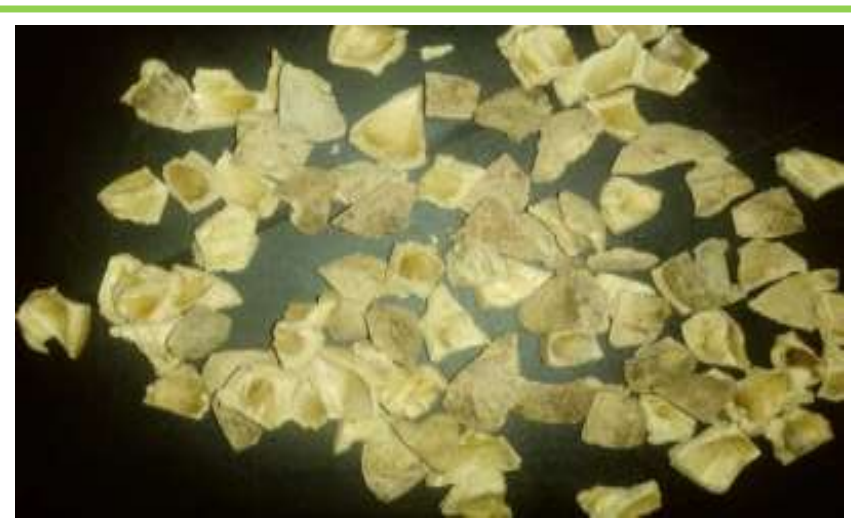

Figura 5. Blanco o testigo partículas de tamaño irregular

\section{Molienda y tamizado del cuesco del cacay}

Empleando la ley de Rittinger de acuerdo a la metodología y como se muestra a continuación. Se determinó el consumo energético durante este proceso (Tabla 5).

Tabla 5. Consumo de energía reducción de tamaño

\begin{tabular}{cc}
\hline $\begin{array}{c}\text { Tamaño de partícula } \\
(\mathbf{m m})\end{array}$ & $\begin{array}{c}\text { Energía reducción de tamaño } \\
(\mathbf{k c a l} / \mathbf{k g})\end{array}$ \\
\hline$\leq 0.25$ & 180.57 \\
$2.36-0.25$ & 14.61 \\
\hline
\end{tabular}

Fuente: Los autores 


$$
\begin{gathered}
E=\frac{p}{m}=K_{R}\left(\frac{1}{d 2}-\frac{1}{d 1}\right) \\
K_{R}=\frac{\frac{p}{m}}{\left(\frac{1}{d 2}-\frac{1}{d 1}\right)} \\
K_{R}=\frac{\frac{0,75 K W}{3,47 K g}}{\left(\frac{1}{0,25 m m}-\frac{1}{10,5 m m}\right)}=0,055 \frac{K W}{K g * m m} \\
E=0,055 \frac{K W}{K g * m m} *\left(\frac{1}{0,25 m m}-\frac{1}{10,5 m m}\right)=0,21 \frac{K W}{K g} \\
0,21 \frac{K W}{K g}=\frac{180,57 \mathrm{kcal}}{\mathrm{kg}} \\
0,017 \frac{\mathrm{KW}}{\mathrm{Kg}}=14,61 \frac{\mathrm{kcal}}{\mathrm{kg}}
\end{gathered}
$$

Seguido a la molienda el producto se sometió a un proceso de tamizaje obteniendo como resultados las fracciones que se muestran en la Tabla 6, permitiendo identificarlas como las de mayor proporción, las cuales se encuentran entre los intervalos de $2.36-0.25 \mathrm{~mm}$ y menores a $0.250 \mathrm{~mm}$.

\section{Secado}

De acuerdo al consumo promedio de un horno como el empleado para el proceso de secado se cuenta con consumo energético de $600 \mathrm{~W} / \mathrm{h}$, entonces la energía consumida por el horno durante el tiempo de secado se muestra en Tabla 7, siendo el consumo energético $\times$ tiempo de secado $600 \mathrm{~W} \times 12 \mathrm{H}$ dando un resultado de $7200 \mathrm{~W} / \mathrm{h}=6190,89 \mathrm{Kcal} / \mathrm{h}$. 
Tabla 6. Resultados del tamizaje

\begin{tabular}{ccccc}
\hline $\begin{array}{c}\text { Tamiz } \\
\text { (apertura) }\end{array}$ & Intervalo (mm) & $\begin{array}{c}\text { Diámetro } \\
\text { promedio }(\mathbf{m m})\end{array}$ & $\begin{array}{c}\text { Total } \\
\text { retenido }(\mathbf{g})\end{array}$ & $\begin{array}{c}\text { Porcentaje } \\
(\%)\end{array}$ \\
\hline $9.5 \mathrm{~mm}$ & $>9.5$ & & 74.62 & 2.14 \\
$3.35 \mathrm{~mm}$ & $9.5-3.35$ & 6.425 & 207.69 & 5.97 \\
$2.80 \mathrm{~mm}$ & $3.35-2.80$ & 3.075 & 114.03 & 3.27 \\
$2.36 \mathrm{~mm}$ & $2.80-2.36$ & 2.580 & 50.33 & 1.44 \\
$0.25 \mathrm{~mm}$ & $2.36-0.25$ & 1.305 & 2577.05 & 74.1 \\
Fondo & $<0.25$ & 0.125 & 453.32 & 13.03 \\
& Total & & 3477.04 & 100 \\
\hline
\end{tabular}

${ }^{*}$ Total obtenido de la sumatoria de 7 ensayos con un promedio de $500 \mathrm{~g}$ cada una.

Tabla 7. Consumo de energía en el proceso de secado

\begin{tabular}{cc}
\hline Nombre de la muestra & Energía secada al horno \\
\hline $\begin{array}{c}\text { T1 y T2 (Secado al horno y tamaño partícula } \\
\leq 0.25 \text { y } 2.36-0.25)\end{array}$ & $6190.89 \mathrm{Kcal} / \mathrm{h}$ \\
\hline
\end{tabular}

Fuente: Los autores

\section{Determinación del poder calorífico superior}

En la Tabla 8 se observan los valores obtenidos del poder calorífico de cada una de las muestras analizadas, donde se incluye el promedio entre los triplicados de cada una de las muestras, así como las medidas de tendencia central que nos permiten establecer que la dispersión entre los triplicados de cada una de las muestras no es significativa, de igual manera en la Tabla 9 se evidencia un consolidado de los promedios de cada muestra. 
Tabla 8. Poder calorífico de las muestras analizadas

\begin{tabular}{cccccccc}
\hline \multirow{2}{*}{$\begin{array}{c}\text { Nombre } \\
\text { de la } \\
\text { muestra }\end{array}$} & \multicolumn{2}{c}{ Poder calorífico (kcal/kg) } & Promedio & Varianza & $\begin{array}{c}\text { Desviación } \\
\text { estándar }\end{array}$ & $\begin{array}{c}\text { Coeficiente de } \\
\text { variación }\end{array}$ \\
\cline { 2 - 7 } T1 & 4343.0545 & 4393.4484 & 4449.2327 & 4393 & 2820.87 & 53.1119 & $1.2 \%$ \\
T2 & 4608.3231 & 4644.9237 & 4628.9488 & 4629 & 336.70 & 18.3494 & $0.39 \%$ \\
T3 & 4188.6033 & 4195.2495 & 4177.0376 & 4189 & 84.93 & 9.2160 & $0.22 \%$ \\
T4 & 4457.8077 & 4465.3735 & 4475.4798 & 4465 & 78.61 & 8.8664 & $0.19 \%$ \\
Blanco & 4075.2543 & 4056.3548 & 4089.2476 & 4075 & 272.49 & 16.5072 & $0.40 \%$ \\
\hline
\end{tabular}

Fuente: Los autores

\section{Balance total de energía}

En la tabla 10 se presenta el balance de energía que se desarrolló de acuerdo a la metodología donde se tuvo en cuenta la energía consumida en cada uno de los pretratamientos a los cuales se sometieron las muestras y los valores de poder calorífico obtenidos de cada una de las muestras.

Tabla 9. Poder calorífico de acuerdo al tamaño de la partícula

\begin{tabular}{cccc}
\hline $\begin{array}{c}\text { Nombre de } \\
\text { la muestra }\end{array}$ & $\begin{array}{c}\text { Tipo de } \\
\text { secado }\end{array}$ & $\begin{array}{c}\text { Tamaño de partícula } \\
(\mathbf{m m}) \mathbf{N}\end{array}$ & $\begin{array}{c}\text { Poder calorífico promedio } \\
\text { (kcal/kg) }\end{array}$ \\
\hline T1 & Horno & $\leq 0.25$ & 4393 \\
T2 & Horno & $2.36-0.25$ & 4629 \\
T3 & Sol & $\leq 0.25$ & 4189 \\
T4 & Sol & $2.36-0.25$ & 4465 \\
\hline
\end{tabular}

Fuente: Los autores 
Tabla 10. Balance energético de los tratamientos

\begin{tabular}{|c|c|c|c|c|c|}
\hline \multirow{2}{*}{$\begin{array}{c}\text { Nombre de la } \\
\text { muestra }\end{array}$} & \multicolumn{3}{|c|}{ Energía consumida (kcal/kg) } & \multirow{2}{*}{$\begin{array}{c}\text { Poder } \\
\text { calorífico } \\
\text { (kcal/kg) }\end{array}$} & \multirow{2}{*}{$\begin{array}{l}\text { Balance } \\
\text { (kcal/kg) }\end{array}$} \\
\hline & Secado & Molienda & Total & & \\
\hline T1 & 6190.89 & 180.57 & 6371.46 & 4393 & -1978.46 \\
\hline $\mathrm{T} 2$ & 6190.89 & 14.61 & 6205.5 & 4629 & -1576.5 \\
\hline T3 & - & 180.57 & 180.57 & 4189 & 4008.43 \\
\hline $\mathrm{T} 4$ & - & 14.61 & 14.61 & 4465 & 4450.39 \\
\hline Blanco & - & - & - & 4075 & 4075 \\
\hline
\end{tabular}

Fuente: Los autores

\section{Análisis de varianza}

El tratamiento estadístico de análisis de varianzas demostró por medio del método HSU (método de comparaciones múltiples) que el tratamiento con mayor poder calorífico se presentó en la muestra T2 y a su vez esta presenta diferencias significativas con todas las otras muestras. Así mismo, se determinó por medio del método Dunnett que todas las muestras presentaron diferencias significativas a favor con respecto al blanco. Por otra parte, el método Tukey comprobó que la muestra T2 obtuvo los mayores niveles y que presenta diferencias significativas con respecto al blanco, así como que las muestras T1 y T4 no presentan diferencias significativas entre ellas. En la Gráfica 1 se muestran los diagramas de caja de cada una de las muestras incluyendo el blanco.

\section{Molienda y tamizado del cuesco del cacay}

Según los resultados obtenidos, el consumo energético del proceso de molienda para los tamaños de partícula comprendidos en el intervalo $<0.25 \mathrm{~mm}$ fue de 180.57 kcal representado un gasto mucho mayor respecto al consumo de energía empleado para la obtención de las partículas comprendidas en el intervalo de 2.36$0.25 \mathrm{~mm}$, de acuerdo a lo expresado por la ley de Rittinger debido a que el tamaño 
de partícula es inversamente proporcional a la energía requerida, por lo que se infiere que a menor diámetro mayor consumo energético.

Además, se observa que las partículas de tamaños superiores alcanzaron un poder calorífico mayor en cada uno de sus tipos de secado, resultado que también se presenta en la investigación realizada por Ahumada et al., (2016) donde se concluyó que entre mayor granulometría mayor poder calorífico.

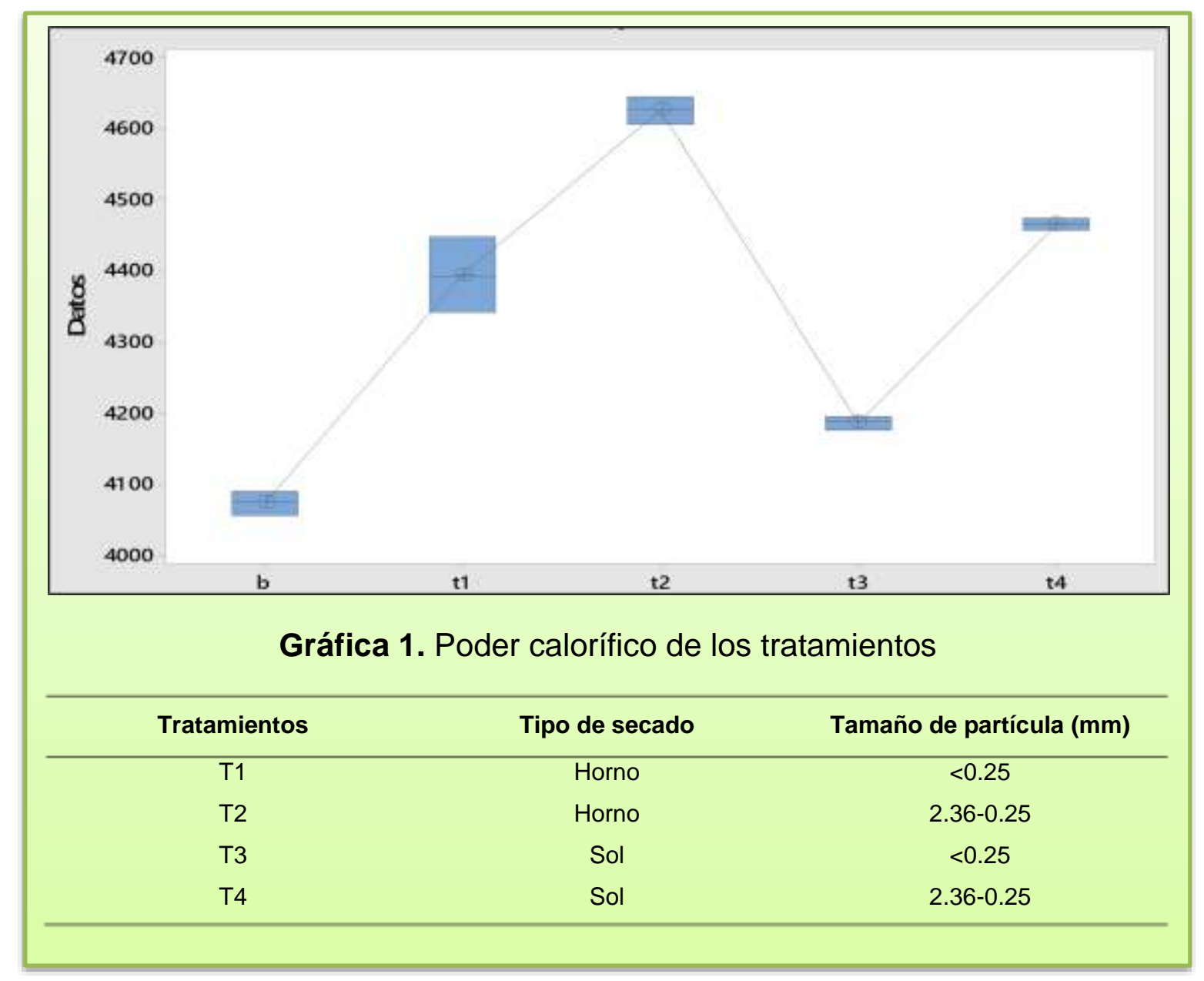

\section{Secado}

El método de mayor eficiencia fue el secado al sol, ya que, se alcanzaron en promedio temperaturas cercanas a los $32^{\circ} \mathrm{C}$ sin requerir un consumo de energía adicional, por otra parte, la baja eficiencia del horno se debe a su alto consumo energético, debido a que es un equipo que alcanza mayores temperaturas, pero que 
requiere a su vez, de un consumo energético elevado. Una vez obtenidos los resultados se observó que las muestras sometidas al secado al sol T3 y T4 alcanzaron valores de 4189 y $4465 \mathrm{Kcal} / \mathrm{kg}$ respectivamente y las muestras sometidas al secado al horno T1 y T2 alcanzaron valores de 4393 y $4629 \mathrm{Kcal} / \mathrm{kg}$ respectivamente (Gráfica 2), aunque los valores obtenidos por el secado al sol son menores en comparación con los obtenidos por el secado al horno, el secado al sol no requiere ningún gasto energético, mientras que el secado al horno demando un gasto energético de $6190.89 \mathrm{Kcal} / \mathrm{kg}$ convirtiéndolo en el menos eficiente.

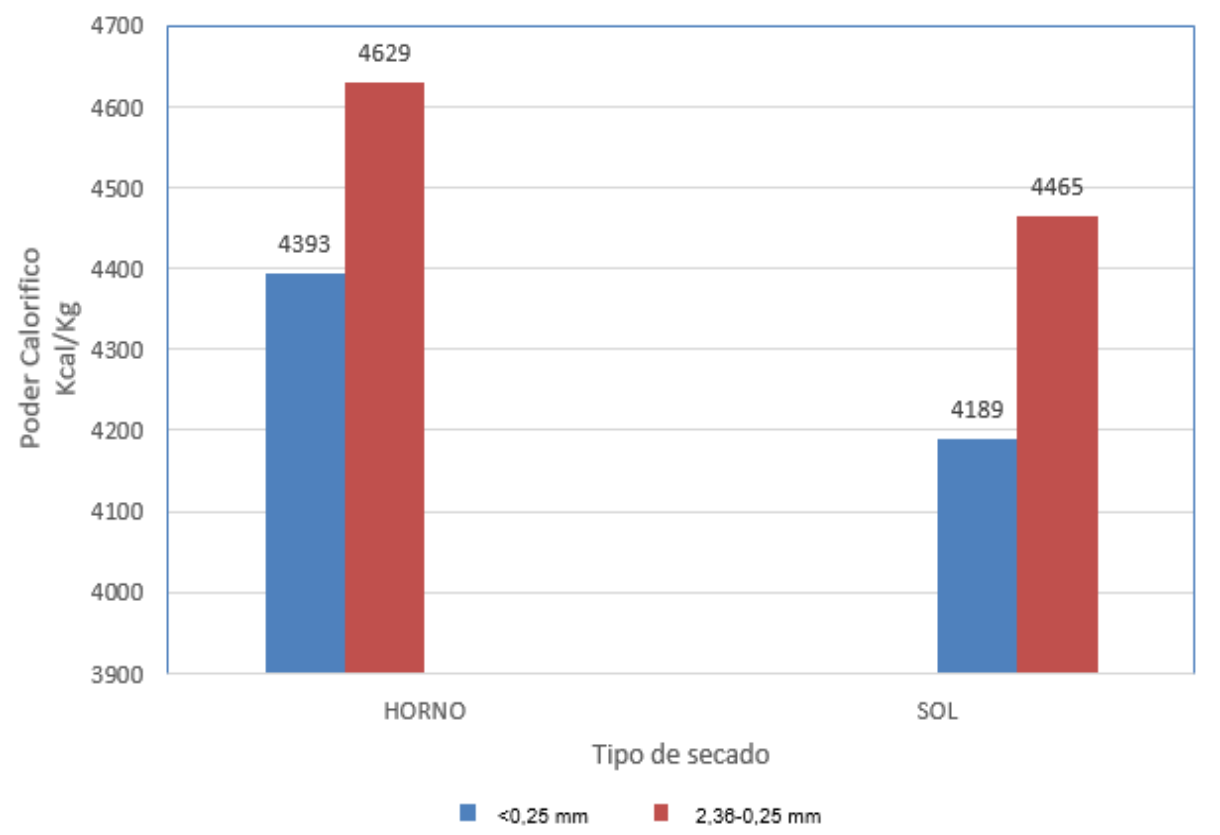

Gráfica 2. Poder calorífico de acuerdo a los pretratamientos

\section{Poder calorífico y comparación con otras biomasas}

De acuerdo a los resultados del poder calorífico de las muestras, este material es capaz de producir una cantidad de energía por kilogramo de sustancia con valores de 4393, 4629, 4189, 4465 y $4075 \mathrm{Kcal} / \mathrm{Kg}$, para las muestras T1, T2, T3, T4 y el Blanco, respectivamente, como se muestra en la Gráfica 3.

Así mismo alcanzó un potencial energético superior al de otros residuos agrícolas como; el bagazo de la caña, la cascarilla de arroz, y el cuesco de la palma de aceite, los cuales registran valores de 1823, 3281 y $3558 \mathrm{Kcal} / \mathrm{kg}$ respectivamente, 
indicando que este residuo es una fuente potencial de energía alternativa y que su uso como biocombustible contribuye a evitar su acumulación.

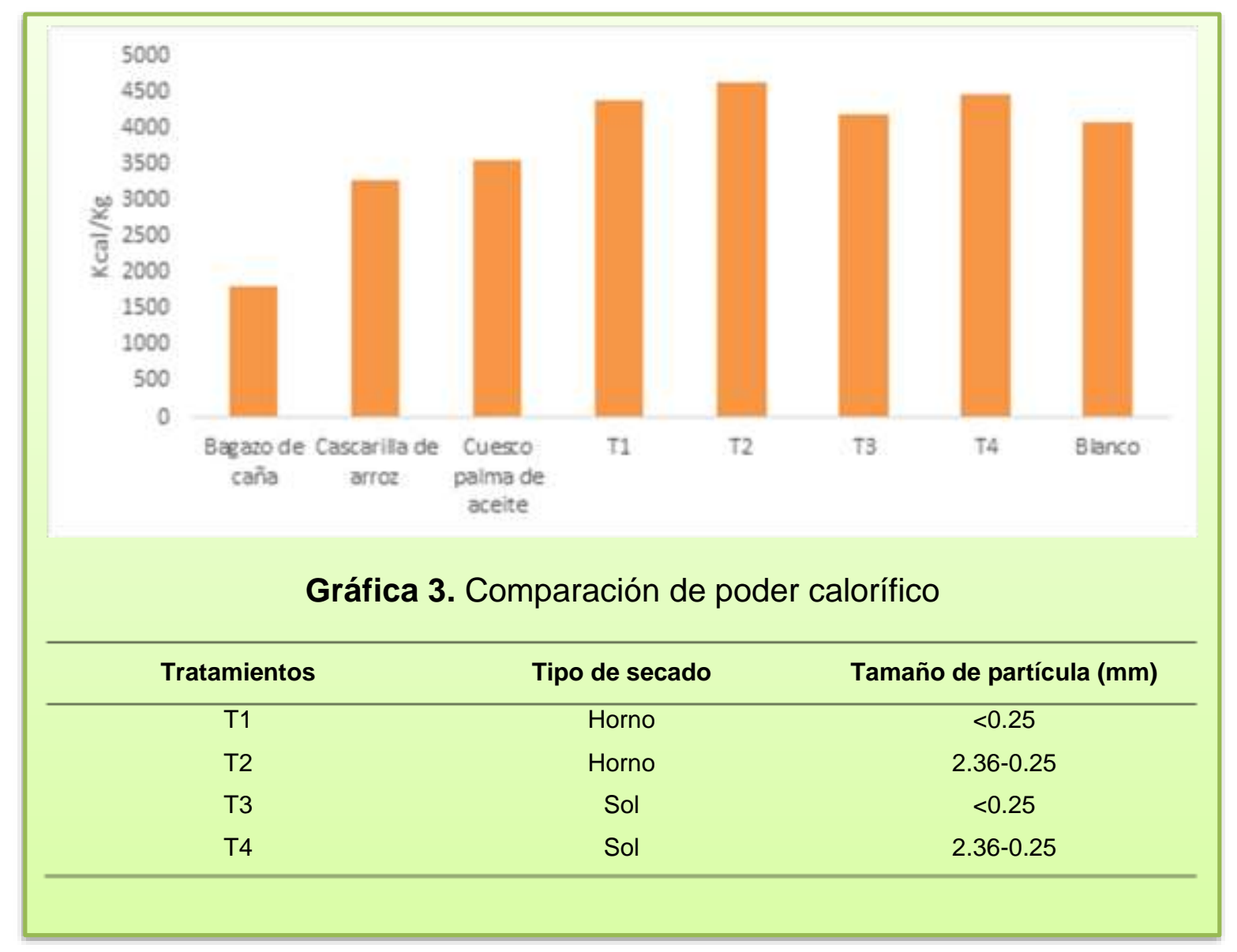

Uno de los factores que inciden en un elevado poder calorífico, según Burschel et al., (2003) es la humedad, quien afirma que la humedad posee un efecto lineal inversamente proporcional con el poder calorífico de los materiales. Efecto que se logra comprobar al observar la diferencia del poder calorífico obtenido a partir de los dos métodos de secado, donde el secado al horno presentó mayores valores de poder calorífico que el secado al sol, ya que, durante el secado al horno el material está expuesto a temperaturas más elevadas, permitiendo una mayor pérdida de humedad. Otro factor decisivo en la capacidad calorífica de los materiales es su composición, puesto que este depende de sus características químicas, así como lo señala Garrués, (2010) en su libro energía de la Biomasa, y de igual manera 
según Okoroigwe y Saffron, (2012) el poder calorífico tiene una relación directamente proporcional con el contenido de carbono fijo.

\section{Balance energético}

Una vez obtenidos los resultados se determinó que la muestra T2 presentó los valores más altos de poder calorífico alcanzando $4629 \mathrm{Kcal} / \mathrm{kg}$ como se logra evidenciar en la Gráfica 1, sin embargo, esta muestra requirió en sus pretratamientos de secado al horno y molienda un consumo de energía de 6205.5 $\mathrm{Kcal} / \mathrm{kg}$ mucho mayor a la energía obtenida, lo que la convierte a su vez en la muestra con mayor gasto energético, por otra parte la muestra con mayor eficiencia entre el consumo de energía de sus pretratamientos y la energía generada fue la muestra T4 la cual alcanzo un poder calorífico de $4465 \mathrm{Kcal} / \mathrm{kg}$ consumiendo 14.61 $\mathrm{Kcal} / \mathrm{kg}$ en sus pretratamientos de secado al sol y de molienda como se puede observar en la Tabla 10.

\section{CONCLUSIONES}

El cuesco de Cacay es capaz de alcanzar altos valores energéticos en comparación con otros residuos agroindustriales tradicionales como el bagazo de la caña, la cascarilla de arroz y el cuesco de la palma de aceite, sin necesidad de emplear algún pretratamiento.

La implementación de estos pretratamientos (molienda y secado) no presentan viabilidad económica exceptuando el secado al sol, sin embargo, los valores más elevados de poder calorífico independientemente del tipo de secado se obtuvieron en las muestras con mayor granulometría.

La muestra T4 logró un aumento del 10\% del poder calorífico con respecto al blanco solo sometiéndose al secado al sol y a la reducción de tamaño con la mayor granulometría, lo que indica que los pretratamientos de esta muestra son los más óptimos para aumentar su poder calorífico.

Teniendo en cuenta la capacidad energética del cuesco de cacay sin ningún pretratamiento se puede sugerir la aplicación de este material como un 
biocombustible alternativo evitando así su acumulación y aprovechando su buen poder calorífico, ya que superó a otras biomasas residuales que se emplean con esta finalidad, abriendo la posibilidad de darle un valor agregado a este material mediante su transformación en un biocombustible

\section{REFERENCIAS BIBLIOGRÁFICAS}

1. Ahumada et al. Optimización de las condiciones de operación de la microgasificación de biomasa para producción de gas de síntesis. 2016. Disponible En: https://scielo.conicyt.cl/pdf/infotec/v27n3/art17.pdf

2. ASTM International, ASTM D-5865-04. Standard test method for the gross calorific value of coal and coke. 2004.

3. Burschel et al. Leña: una fuente energética renovable para Chile. 2003. Disponible https://books.google.com.co/books?id=RwIVY96BUuQC\&pg=PA70\&dq=de+qu e+depende+el+poder+calorifico\&hl=es\&sa=X\&ved=0ahUKEwjah 7lht3hAhXIx FkKHbHeDm0Q6AEIKDAA\#v=onepage \&q=de\%20que\%20depende $\% 20 \mathrm{el} \% 20$ poder\%20calorifico\& $\mathrm{f}=$ false

4. Contexto ganadero. El Cacay Inchi, una alternativa de desarrollo sostenible en Colombia [en línea]. En: Contexto ganadero. 08 de Julio 2013. Recuperado 20 sep. 2018. Disponible En: http://www.contextoganadero.com/blog/el-cacayinchi-una-alternativa-de-desarrollo-sostenible-en-colombia

5. Durango R., Oquendo J. Caracterización y evaluación energética de biocombustibles solidos elaborados a partir de biomasa agroindustrial y carbón mineral del departamento de Córdoba. Universidad de Córdoba, 2016.

6. Garrués J. Energía de la Biomasa (volumen I). Universidad de Zaragoza, $415 \mathrm{p}$, 2010. Recuperado 24 Abril 2019. Disponible En: https://books.google.com.co/books?id=P58rcPu5090C\&pg=PA415\&dq=Otro+f actor+decisivo+en+la+capacidad+calor\%C3\%ADfica+de+los+materiales+es+s u+composici\%C3\%B3n\&hl=es\&sa=X\&ved=0ahUKEwiEoJWJxOThAhXatlkKH U5eBhMQ6AEIMzAC\#v=onepage\&q=Otro\%20factor\%20decisivo\%20en\%20la \%20capacidad\%20calor\%C3\%ADfica\%20de\%20los\%20materiales\%20es\%20 su\%20composici\%C3\%B3n\&f=false

7. Gutiérrez E., Ulloa A. Reducción de tamaño. Recuperado 26 Septiembre 2018. Disponible En: https://es.slideshare.net/FanychanCosplayer/reduccion-detamao

8. Herrera A.C. et al. Densidad, composición química y poder calorífico de la madera de tres especies de encinos (Quercus candicans, Q. laurina y Q. rugosa). Ciencia Nicolaita, [S.I.], n. 72, 2018. Recuperado 29 Septiembre 2018. Disponible En: https://www.cic.cn.umich.mx/index.php/cn/article/view/345

9. Okoroigwe E., Saffron, C. Determination of bio-energy potential of palm kernel shell by physicochemical characterization. Nigerian Journal of Technology, 31 (12): 329-336. 2012.

10. Peñaranda L. V., Montenegro S. P., Giraldo, P. A. Aprovechamiento de residuos agroindustriales en Colombia. Revista de Investigación Agraria y Ambiental, 
[S.I.], 8 (2): 141-150. 2017. Recuperado 20 sep. 2018. Disponible En: http://hemeroteca.unad.edu.co/index.php/riaa/article/view/2040/2251

11. Procolombia. Cacay, la nuez colombiana para cosméticos que fascina al mundo [en línea]. En: PROCOLOMBIA. 06 de noviembre 2014. Recuperado 20 Septiembre 2018. Disponible En: http://www.procolombia.co/noticias/cacay-lanuez-colombiana-para-cosmeticos-que-fascina-al-mundo

12. Redacción El Tiempo. El promisorio cultivo de cacay en la amazorinoquia [en línea]. En: el Tiempo. 01 de febrero 2017. Recuperado Fecha de acceso: 20 sep. 2018. Disponible En: https://www.eltiempo.com/colombia/otrasciudades/cacay-33229 